\title{
Identifying rotated letter-like symbols
}

\author{
MALCOLM G. ELEY \\ University of Tasmania, Hobart, Tasmania 7001, Australia
}

\begin{abstract}
Previous research has shown that the identification of rotated alphanumeric symbols seems to be performed via the extraction of critical features encoded invariant to the symbol's orientation. The present research argued that the use of such feature extraction processes might be a function of, first, a subject's familiarity with the symbols, and second, the number of symbols from which a presented symbol is sampled. Earlier research has used highly overlearned alphanumerics, in sets of six symbols; this practice is argued here as being seemingly conducive to feature extraction. In two experiments, the generality of a feature extraction interpretation, in contrast to one of mental rotation, was tested by having subjects previously trained to relative high- vs. low-familiarity criteria identify novel symbols in conditions in which a presented symbol was 1 of either 5 or 20 possibilities. Identification response times were found to be constant across all nonstandard orientations of presented symbols, irrespective of symbol familiarity or symbol set size. The findings support the generalization of a feature extraction interpretation to varying numbers of novel symbols of varying familiarity.
\end{abstract}

When an individual verifies a visual symbol that has been rotated away from its standard upright orientation, response times are typically found to be longer the greater is the variation from that normal orientation. These findings have been demonstrated for "normal" vs. "mirror image" judgments of alphanumeric symbols (Cooper \& Shepard, 1973) and of two- or four-sided figures (Petrusic, Varro, \& Jamieson, 1978), for "same" vs. "different" judgments of randomly generated twodimensional shapes (Cooper \& Podgorny, 1976) and of two-dimensional representations of three-dimensional objects (Tapley \& Bryden, 1977), and for whether a probe figure is a subcomponent of a criterion figure vs. a mirror image of a subcomponent (Pylyshyn, 1979).

The explanation given for such findings is that prior to the verification decision, the stimulus to be judged is mentally rotated back to a standard orientation and then matched against some criterion. This explanation has been found to hold both when the criterion against which the judgment is to be made is a physically present visual stimulus (Petrusic et al., 1978; Pylyshyn, 1979) and when it is an internal memorial representation (Cooper \& Podgorny, 1976; Cooper \& Shepard, 1973). In that the "rate of mental rotation" has been found to reflect the complexity of stimuli in the former case, it would seem that there may be some variation in what is actually rotated prior to the verification decision, but the proposal that test stimuli are nonetheless mentally rotated remains reasonable.

Corballis, Zbrodoff, Shetzer, and Butler (1978)

The research reported here was supported by a grant from the Recurrent Research Funds of the University of Tasmania. The author's address is Department of Educational Studies, University of Tasmania, G.P.O. Box 252C, Hobart, Tasmania 7001, Australia. recently investigated whether mental rotation played a role in the identification (as opposed to verification against some criterion) of rotated alphanumeric symbols. Their subjects were presented a symbol or its mirror version at one of six orientations and were required simply to say that symbol's label. The lack of a consistent relationship between identification response times and angle of orientation was interpreted as evidence that "identification does not normally require mental rotation" (Corballis et al., 1978, p. 101). Rather, their data seemed to support the notion that identification is a function of the extraction of feature information that is independent of the stimulus' orientation.

However, as Corballis et al. (1978) themselves recog. nized, these findings on identification may be restricted in their generality. It is well-known that the processing strategies employed by subjects in a variety of verificationtype tasks are quite often flexible and sensitive to alterations in task demands (Cooper, Note 1). For instance, in verifying sentences against diagrams, the provision of processing time between sentence and diagram presentations (Glushko \& Cooper, 1978) and the presentation of sentences in aural vs. written modes (Eley, 1981) are both variables that have been found to influence processing strategy. Indeed, in matching random shapes against a criterion, subjects have even been found to exhibit between-subjects variation in processing strategy within the same set of task presentation conditions (Cooper, 1976). It would seem, then, entirely reasonable that the generality of the Corballis et al. (1978) symbol identification findings would need to be explicitly tested. The purpose of the present research was to contribute to such a test.

Intuitively, two variables would seem to be of potential influence on strategies of rotated symbol identification. The first is familiarity. In recent research on mental 
size comparisons, processing strategies were found to be sensitive to the degree of prior overlearning with task stimuli (Kosslyn, Murphy, Bemesderfer, \& Feinstein, 1977). In particular, size disparity effects (faster comparisons for more disparate items, typically interpreted as evidencing analogue processing) were found to be lessened when subjects were given extensive prior overlearning in assigning task stimuli to size categories. The interpretation favored was that overlearning improved the accessibility, and thus speed of retrieval, of the discretely encoded category membership information and thus made it more likely that across-category size comparisons would be decided via discrete rather than analogue processes.

A similar argument might be applied to rotated symbol identifications. As noted previously, the Corballis et al. (1978) findings are consistent with identification occurring via the extraction of features. Such a processing strategy would seem to require, however, that whichever features served as the bases for identification, they would need to be sufficiently well learned as to be employable irrespective of symbol orientation. Should these defining features be not so learned, and thus not accessible irrespective of orientation, it might be more efficient, and thus more attractive, for a subject to mentally rotate the test symbol for matching against some memorial representation of a labeled standard criterion. Since such representations would not need to be encoded invariant to symbol orientation, they could be functional at a lower level of symbol familiarity than could features that were.

The suggestion, then, is that at higher levels of symbol familiarity, critical features, encoded invariant to orientation, would be sufficiently accessible for feature extraction processes to be the bases of efficient identification. However, at lower levels of familiarity, only representations of symbols in their standard upright orientations might be accessible. In such instances, the more efficient strategy of symbol identification could then be via mental rotation processes. In that the Corballis et al. (1978) work employed highly familiar alphanumeric symbols, stimuli that would favor identification via feature extraction, it could be argued that their findings were only to be expected and that the role of symbol familiarity has not been tested.

The second intuitively influential variable concerns the size of the set of symbols that constitute identification possibilities. If a test symbol is one of only a small number that could be presented for identification, then the requirement of a feature extraction strategy that the critical features of all symbols be well learned and accessible to retrieval would be expected to engender only minimal processing demands. However, as the number of symbols that could be presented increases, so also must the number of critical features that would need to be accessibly encoded. It is conceivable that too large a number of potential test symbols could involve such a processing load, with the necessity for maintaining a large set of features encoded invariant to orientation, that a feature extraction strategy might prove relatively cumbersome.

In contrast, a mental rotation strategy should require only that representations of symbols in their standard orientations be encoded and accessible. If it can be assumed that the lack of any necessity for orientation invariance would mean that such representations could be simpler than those that were orientation invariant, then increased numbers of symbols might exact a lesser toll on the encoding and retrieval processes within a mental rotation strategy than within a feature extraction strategy. In brief, then, with small numbers of symbols sampled, identification by feature extraction would likely be faster and more efficient than identification by mental rotation, in that no mental manipulation of the symbol is required prior to the identification response. However, with larger numbers of symbols, the increased processing demands on a feature extraction strategy could render it slower overall compared with mental rotation and thus prompt a subject to use the latter instead.

Corballis et al. (1978) employed only six alphanumeric symbols in their experiments. It could thus be argued that such a small number of symbols would have favored the feature extraction strategy that they evidenced over some sort of mental rotation strategy. In summary, if the Corballis et al. findings can be shown to generalize to the identification of symbols drawn from sets of varying size and, further, to that of symbols of varying familiarity, then those findings will be considerably strengthened.

\section{EXPERIMENT 1}

This first experiment sought to test the generality of the Corballis et al. (1978) findings that mental rotation was not normally required in the identification of rotated symbols. Specifically, the robustness of these findings was tested with respect to the two variables discussed above: symbol familiarity and identification set size. A set of novel letter-like symbols was generated and then labeled with CVC trigrams balanced for meaningfulness. Symbol familiarity was varied by having subjects learn to label the symbols to either of two performance criteria. Set size was varied by having subjects subsequently perform rotated symbol identifications on either the entire set or a subset of the generated symbols.

\section{Method}

Subjects. Twenty-eight staff and student volunteers were randomly allocated to four groups, each comprising three males and four females.

Stimulus materials. Twenty letter-like symbols (five were taken from those of Gibson, Gibson, Pick, \& Osser, 1962) were generated to match, proportionately, the complexity of the 
Roman capital alphabet (see Figure 1). These were then randomly allotted to four unique subsets of five symbols each such that each subset had one symbol from each of Symbols 1-4, $5-8,9-12,13-16$, and 17-20. This procedure ensured that the four resulting subsets were equivalent in terms of symbol complexity.

Twenty CVC trigrams were selected from Archer's (1960) scaling within the meaningfulness range of 60-70 inclusive (see Figure $1 \mathrm{also}$ ) such that there were four instances of each of the vowels $A, E, I, O$, and $U$ and such that there were two instances of each of the initial consonants $B, D, G, K, M, N, P, R, S$, and T. Selection was further constrained in that (1) all CVCs were pronounceable, (2) silent consonants were excluded, (3) repeat consonant CVCs were excluded, (4) there were different final consonants for CVCs having the same initial consonant and for CVCs having the same middle vowel, and (5) trigram words were excluded, as were homophones of words. These 20 trigrams were then randomly allocated as labels to the symbols such that within each five-symbol subset, each label had a different initial consonant and a different middle vowel. The resulting subsets were Symbol-Label Pairs 2-7-11-16-20, 4-5-10-14-19, 3-6-12-1317, and 1-8-9-15-18.

Each symbol was photographed onto 110 -size transparencies at orientations of $0 \mathrm{deg}$ (standard upright), $60 \mathrm{deg}$ (clockwise), $120 \mathrm{deg}, 180 \mathrm{deg}, 240 \mathrm{deg}$, and $300 \mathrm{deg}$. The symbols were shown in black line, centered on a white circular background surrounded by black. Each symbol had a small dot that designated the top of the symbol. The labels were also photographed onto transparencies with the same circular layout format as the symbols.

The 20 symbol-label pairs, with standard upright orientations, were ordered into three random sequences for use as

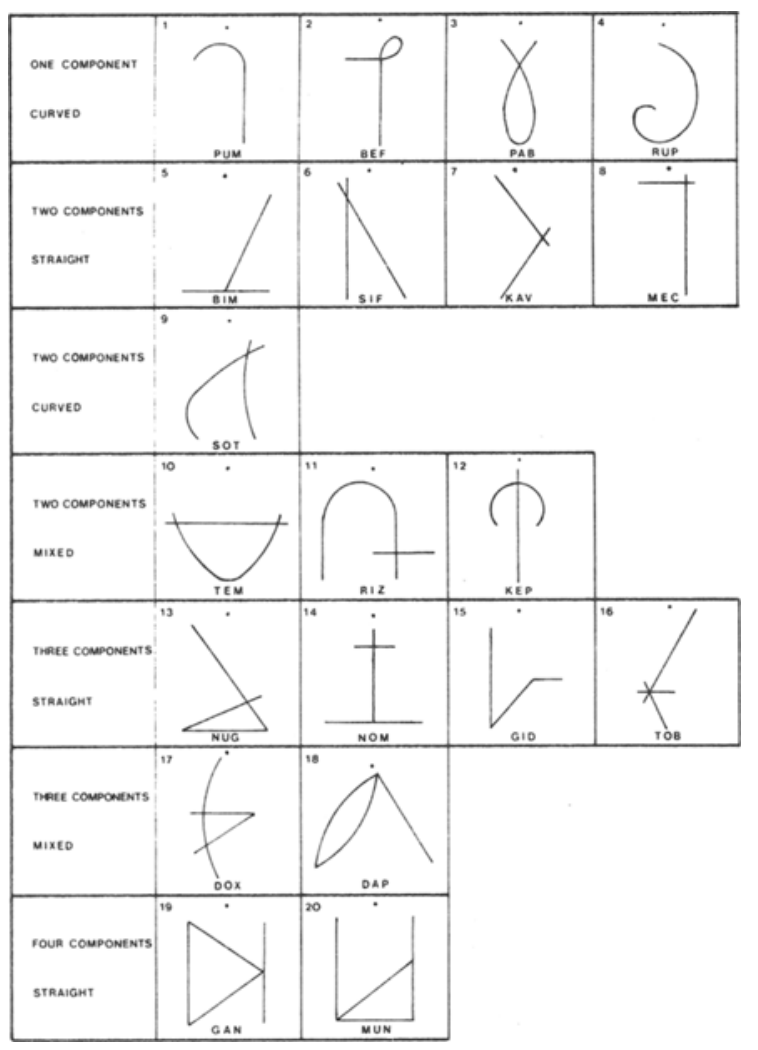

Figure 1. Symbols and labels used in Experiments 1 and 2. training blocks. Also for use in the training, the 20 standard symbols and their labels were laid out in random sequence on a $20 \times 24 \mathrm{~cm}$ card. The 20 symbols at each of the six orientations were randomly ordered into four 30-trial experimental blocks such that each symbol occurred either once or twice in each block and such that each orientation occurred five times in each block. Similarly, four 30-trial experimental blocks were constructed for each five-symbol subset such that each symbol occurred once at each orientation in each block.

Procedure. All subjects were initially trained to label all 20 symbols. A subject first studied the card showing each symbol with its label for $2 \mathrm{~min}$. The subject was then presented one of the 20-trial training blocks. In each trial, a single symbol was projected onto a rear-projection screen, where it remained in view while the subject attempted to recall its label. Following this recall attempt, the label was projected as feedback. There were $5-\mathrm{sec}$ rest delays between training trials.

A subject continued through such study-plus-training-block cycles until a criterion of either two or five consecutive allcorrect training blocks had been achieved. Subjects were thus trained to either lower or higher levels of familiarity with the symbols.

Following the training, each subject was administered four 30-trial experimental blocks. In each trial, a subject was presented a single symbol at one of the six orientations and was required to say its label. On each trial, a .5 -sec warning tone occurred $.5 \mathrm{sec}$ before the symbol's onset, the symbol remained present until after the subject's response, and there was a 5 -sec delay from the symbol's removal to the start of the next trial. Subjects responded vocally, and their responses were timed (via a voice-operated relay) from the onset of the symbol. All subjects were run individually, and they were instructed to respond as quickly as they could consistent with accuracy.

Half of the subjects trained to each of the criteria were administered experimental blocks in which any of the $20 \mathrm{sym}$ bols could be presented in a trial, and the remaining subjects were administered blocks in which the presented symbol was always from one of the five-symbol subsets. For subjects in this five-symbol condition, the subsets from which their experimental blocks were derived were counterbalanced across subjects. Further, the order of presentation of the experimental blocks was also counterbalanced across subjects for both the 5- and 20-symbol conditions. Each subject was thus trained to one of two levels of familiarity with the total symbol set and was then administered 120 experimental trials, each of which required the labeling of a symbol that could be either 1 of 20 or 1 of 5 . These conditions defined the four experimental groups.

\section{Results and Discussion}

The number of training cycles attempted by a subject before achieving the first errorless cycle was entered into a one-factor analysis of variance on the four groups. It proved nonsignificant $[\mathrm{F}(3,24)<1]$, indicating that the assumption that equivalent groups had resulted from the random allocation of subjects was reasonable.

The identification response times from the first experimental block were discarded as practice. For the remaining three blocks, mean response times were calculated for each symbol orientation within each block for each subject. These means were calculated by averaging the four fastest response times of the five recorded for a subject at each orientation within each block. The latter procedure was intended to control for any overly long response times resulting from wavering attention and other extraneous distractions. These mean 
response times were entered into an analysis of variance with the factors of training criterion (two vs. five errorless cycles), symbol set ( 5 vs. 20 symbols sampled), block (Experimental Blocks 2, 3, and 4), and symbol orientation $(0,60,120,280,240$, and $300 \mathrm{deg})$. Errors were not analyzed due to their very low frequencies: $.87 \%$ over the three analyzed blocks. The group mean response times for each orientation for each experimental group are shown in Figure 2.

Neither the training criterion main effect $[F(1,24)<1]$ nor the Training Criterion by Symbol Set interaction $[F(1,24)=1.26, p<.30]$ proved significant, but the symbol set main effect was found to be reliable $[F(1,24)=48.88, p<.00001]$. Thus, it would seem that while there were no reliable differences between the response times of subjects trained to either of the two training criteria, subjects who had to identify a symbol that was 1 of 5 possibilities could do so far quicker than those for whom the symbol was 1 of 20 possibilities.

The blocks main effect proved significant $[F(2,48)=$ $9.77, \mathrm{p}<.001]$, as did also, albeit marginally, the Blocks by Symbol Set interaction $[F(2,48)=3.81$, $\mathrm{p}<.05$; but for the more conservative Greenhouse and Geisser df of $1,24, p<.10]$. Response times for earlier experimental blocks were thus slower than those for later, and the decreases in response times across blocks tended to be more pronounced for the 20-symbol subjects than for the 5-symbol subjects.

Both the symbol orientation main effect $[F(5,120)=$ $3.69 ; \mathrm{p}<.01$; although for the conservative Greenhouse and Geisser df of $1,24, p<.10$ ] and the Orientation by

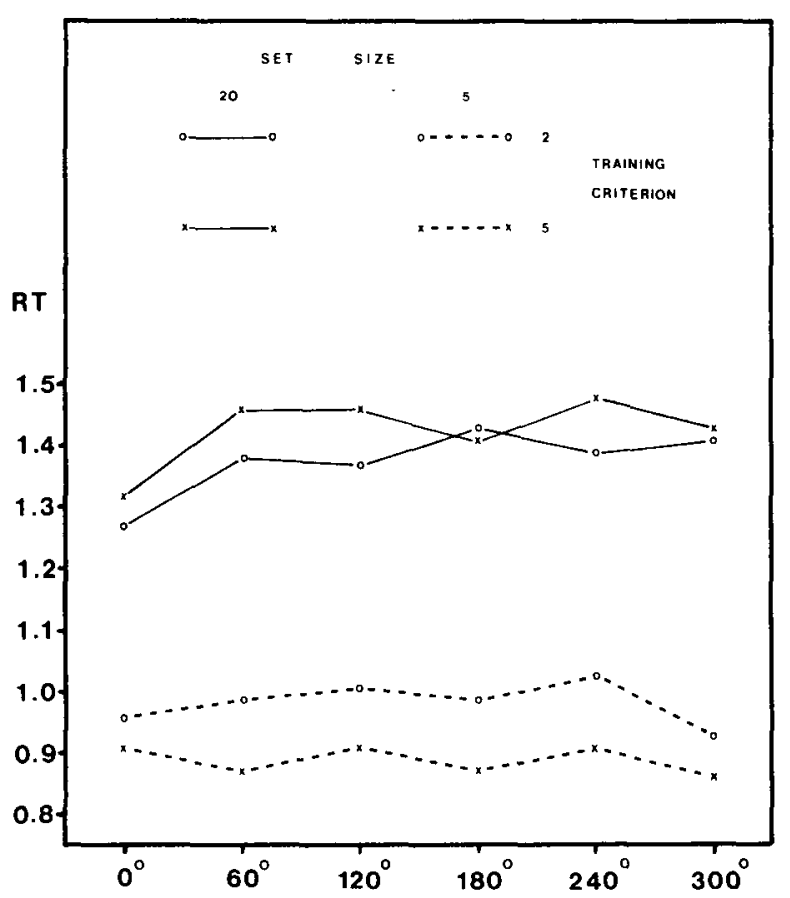

Figure 2. Mean identification response times (in seconds) pooled over Blocks 2, 3, and 4 for each orientation angle.
Symbol Set interaction $[F(5,120)=2.83, p<.05$; but with more conservative $\mathrm{df}$ of $1,24, \mathrm{p}<.25$ ] showed some tendency toward reliability. Simple tests on the interaction showed that while the orientation effect was significant for Symbol Set Size $20[F(5,120)=5.23$, $\mathrm{p}<.01]$, it was not for Size $5[F(5,120)=1.29, \mathrm{p}<.5]$. Duncan's new multiple-range tests showed the former simple test to be due to the 0 -deg response time's being less than those for each of the other orientations, with those others being not reliably different from each other $(p<.05)$. Thus it would seem that the appropriate interpretation of this main effect and the interaction would be that irrespective of whether a subject had to identify a symbol that was 1 of 5 or of 20 possibilities, there were no reliable differences between the identification response times for symbols presented at any of the nonstandard orientations. The one qualification to this interpretation would be that when the presented symbol was 1 of 20 possibilities, there was a tendency for response times to be faster for the standard 0-deg orientation. None of the remaining effects in the overall analysis was significant.

The issue of concern in the present experiment was whether variations in either the subject's familiarity with the symbols or the number of symbols that could be presented for identification would influence the processing strategies employed. There seem to be two basic possibilities: (1) identification by a process of extracting critical features encoded invariant with respect to symbol orientation and (2) identification by the mental rotation of the presented symbol back to some standard orientation at which it could be matched against memorial representations of each possibility. The efficacy of the first of these two possibilities would seem to depend upon the ready accessibility of the encoded critical features. It was argued, first, that such accessibility might be a function of a subject's familiarity with the symbols to be identified, and second, that as the number of symbols that could be presented increased, so also would the number of critical features that would need to be maintained at high accessibility. The less familiar were the symbols or the greater was the number that could be presented, the more demanding a feature extraction strategy might become and the greater might be the probability that a mental rotation strategy would be adopted. Since the recent work of Corballis et al. (1978) had evidenced feature extraction with small numbers of highly familiar alphanumeric symbols, the present experiment investigated the identification of rotated symbols under variations of both symbol familiarity and symbol set size.

The use of feature extraction would predict that identification response times should be constant across the different symbol orientations. The use of mental rotation, however, would predict that these response times should be slower the greater the deviation of the presented symbol away from the standard upright 
orientation. Clearly, the findings of the present experiment are in accord with subjects' having used feature extraction under each of the familiarity by symbol set size conditions. There was no indication in the data of any tendency for the response times to vary in sympathy with a symbol's deviation from the standard. Within each of the four experimental groups, there were no significant differences between the response times for symbols presented at any of the nonstandard orientations. The finding that for subjects in the 20 -symbol condition, response times for the 0-deg orientation were faster than any of the others is perhaps more parsimoniously interpreted as a simple practice effect; the training trials always used standard upright symbols, and thus when these were presented during the later experimental trials, their identification would be more practiced than that of the nonstandard symbols. That the five-symbol subjects did not show a similar 0-deg practice effect can be interpreted as due to their response times' being already quite quick; their response times for all orientations were sufficiently brief as to leave little margin for any 0 -deg practice effect to be manifested.

The differential training criteria failed to produce any significant effect on response times. Not only were the mean response times of subjects trained to two errorless cycles no different from those of subjects trained to five errorless cycles (means of $1.181 \mathrm{sec}$ and $1.158 \mathrm{sec}$ ), but also there was no interaction with orientation $(F<1)$. That is, subjects of both training conditions evidenced feature extraction. Moreover, the present data provided no basis for expecting that any further reduction in familiarity, should such be methodologically realizable, might prompt mental rotation. The correlation between the number of training cycles attempted before achieving the first errorless cycle and the standard deviation of a subject's identification response times on Blocks 2, 3, and 4 was positive (albeit nonsignificant). That is, if anything, there was a tendency for those subjects having had the least exposure to the symbols during training to exhibit the more uniform response times. It would seem, then, that within the present range of variation and with symbols of the type employed here, symbol familiarity might not be an important factor in determining feature extraction vs. mental rotation based strategies of symbol identification.

Symbol set size did influence response times, but not symbol identification strategy. Subjects identifying a symbol that was 1 of 20 possibilities did so significantly slower than those for whom the presented symbol was 1 of 5 possibilities, but both groups exhibited the same feature extraction pattern of response times. For both groups, identification response times were constant across all nonstandard orientations. These findings can be interpreted as showing that increasing the symbol set size from 5 to 20 symbols, and thus increasing the number of critical features that would need to be encoded and maintained at high accessibility, did lead to increased processing demands, but apparently not sufficient to prompt a switch away from a feature extraction strategy. The question still remains, then, as to whether increasing the symbol set size still further might eventually prompt the use of a mental rotation based identification strategy, or indeed whether the symbol set size is important at all in determining strategy.

Two possibilities remain about which the present data allow some comment. First, subjects may have initially attempted mental rotation but, with practice, abandoned it in favor of feature extraction. Such would predict that at least one of the four interactions involving both blocks and orientation should be significant. None was; in fact, only the four-way interaction had an $F>1[F(10,240)=1.44, p<.20]$. Further, an analysis of the practice trials also showed no orientation effect $(F<1)$ and no significant interactions involving orientation. This first possibility thus seems unlikely.

Second, relatively complex symbols could be argued as entailing greater feature encoding, and thus as prompting a greater tendency to opt out of feature extraction than might simple symbols. From the experimental blocks of Experiment 1, mean response times were derived for simple vs. complex symbols (one or two vs. three or four components) at each orientation for each subject. A four-way analysis of variance showed that while responses to simple symbols were faster than those to complex symbols, at least for Symbol Set 5 subjects [interaction $F(1,24)=8.62, p<.01$ ], none of the interaction effects involving both complexity and orientation proved reliable. The three-way interaction with set size was the only effect of the four to have an $F>1[F(5,120)=2.50, p<.05$; but for conservative df of $1,24, p<.25]$, and this was interpretable as a combination of the above Complexity by Set Size interaction with faster 0-deg responses under Set Size 20. In brief, the pattern of response times across symbol orientation did not differ with respect to symbol complexity. If complexity does determine strategy choice, it would seem that it might do so only for symbols of greater complexity than those used here.

In conclusion, then, the findings of the present experiment allow the earlier Corballis et al. (1978) findings to be generalized. Not only does feature extraction seem to be the basis for identifying small numbers of highly familiar alphanumeric symbols, irrespective of their orientation, but it seems also to underlie the identification of varying numbers of novel symbols of varying familiarity, again irrespective of orientation.

\section{EXPERIMENT 2}

Corballis et al. (1978), in investigating the mental processes employed in identifying rotated alphanumeric symbols, deliberately used the same symbols used in 
Cooper and Shepard's (1973) work on the verification of rotated symbols. Such a methodology allowed Corballis et al. to argue that their identification response time findings, which showed a different pattern compared with the verification response times of Cooper and Shepard, evidenced a difference in the mental processes involved in identification as compared with verification, and not just a difference attributable to dissimilar stimuli or experimental procedures. A similar interpretational possibility needs to be dispelled here.

Experiment 1 employed a novel set of stimuli, and it was argued that the findings there supported a generalization of the Corballis et al. (1978) feature extraction findings. However, this argument rests on the assumption that the identification of the novel letter-like symbols used belongs to the same class of tasks that were administered in the Cooper and Shepard (1973) and Corballis et al. experiments. Just as Corballis et al. needed to maintain stimulus comparability with Cooper and Shepard in order that they might argue for an identification vs. verification processing difference, the Cooper and Shepard verification findings need to be replicated with the present symbols in order that the Experiment 1 findings not be interpretable as a simple artifact of the novel symbols used. The purpose of Experiment 2 was to attempt such a replication.

\section{Method}

Subjects. Nine undergraduate students (two male, seven female) served as volunteer subjects.

Stimulus materials. Two sets of five symbol-label pairs were selected at random from the materials of Experiment 1, with the constraints that only those symbols that were asymmetric to both rotation and mirror reflection be sampled, that each set contain one symbol from each of the Groups 1-4, 5-8, 9-12, 13-16, and 17-20 (see Figure 1), and that neither set contain two labels with the same initial consonant. This selection procedure ensured that the two sets were comparable and that both sampled the full range of symbol complexity. The resulting symbol-label pairs were BEF-SIF-RIZ-TOB-MUN and PUMBIM-SOT-GID-DAP.

For each set, three copies of each of the standard 0-deg symbols, together with their appropriate labels, were formed into a 15-trial training block. No two consecutive trials contained the same symbol, and each symbol occurred once (randomly placed) within each of Trials 1-5, 6-10, and 11-15.

Five 24-trial experimental blocks were also formed for each of the two sets. Each block contained four symbols at each of the six orientations: two normal and two mirror reflected. In each block, no symbol occurred more than once at any orientation (irrespective of normal vs. mirrored), and each symbol occurred either four or five times overall. Over the five blocks, all symbols occurred equally often at each orientation and as both normal and reflected versions.

Procedure. All subjects were initially trained to label each of the symbols in their allocated (random) five-symbol set. A subject first studied, for $2 \mathrm{~min}$, a card showing each symbol, together with its label, in random array. The subject was then administered the 15-trial training block, in each trial of which he attempted to recall the label of a presented symbol, postresponse feedback being the presentation of that label. These study-plus-test cycles continued until the subject achieved a criterion of two consecutive all-correct cycles.

Following the training, each subject was administered five 24-trial experimental blocks appropriate to his symbol set. The time plan for each trial was the same as for Experiment 1. However, in the present experiment, half the symbols were mirror reflections, the subject's task was to respond yes/no as to whether a presented symbol was either a normal or a mirrorreflected version, and responses were not vocal but, rather, were made with a two-way toggle switch operated with the subject's preferred hand. The order of presentation of experimental blocks was counterbalanced across subjects. Subjects were instructed to respond as quickly as they could consistent with accuracy.

\section{Results and Discussion}

The response times from the first experimental block were discarded as practice. Errors were not analyzed, due to their very low frequency of $3.36 \%$ over the four analyzed blocks.

For each subject, a mean verification response time was computed for both normal and mirror-reflection verifications at each of the six orientations. These means were computed by averaging over the seven fastest response times of the eight recorded for each normal/reflected by orientation combination over the four analyzed blocks; the slowest times were excluded as a means of controlling for the effects of attentional lapses and other miscellaneous distractions. These response time means were entered into an analysis of variance, the factors being symbol orientation $(0,60$, $120,180,240$, and $300 \mathrm{deg}$ ) and symbol version (normal vs. mirror reflected). Response times were pooled over allocated symbol set, since the reason for having the two sets was to allow both a small symbol set to be used, paralleling Cooper and Shepard (1973), and a wider range of symbols from Experiment 1 to be sampled. The group mean response times are shown in Figure 3.

The symbol version main effect was significant $[F(1,8)=28.91, p<.001]$, with response times being shorter for normal than for mirror-reflected versions. The symbol orientation main effect was significant $[F(5,40)=16.39, p<.00001$; with conservative df of $1,8, p<.011$, with response times being longer the greater was a symbol's deviation away from the standard 0 -deg orientation. The interaction was not significant $[\mathrm{F}(5,40)<1]$.

The findings from the present experiment, then, replicate those of Cooper and Shepard (1973) on the verification of rotated alphanumeric symbols. The time required to verify a symbol as being either normal or mirror reflected would seem to be a function of that symbol's orientation, for both alphanumeric and novel letter-like cases. Importantly for the present research, this replication of Cooper and Shepard's verification findings using the symbols from Experiment 1 indicates that those Experiment 1 findings should not be interpreted as a mere artifact of the novel symbols used. Instead, the Experiment 1 findings can now reasonably be taken as supporting the generalization of the Corballis et al. (1978) feature extraction interpretation to larger sets of nonalphanumeric symbols. 


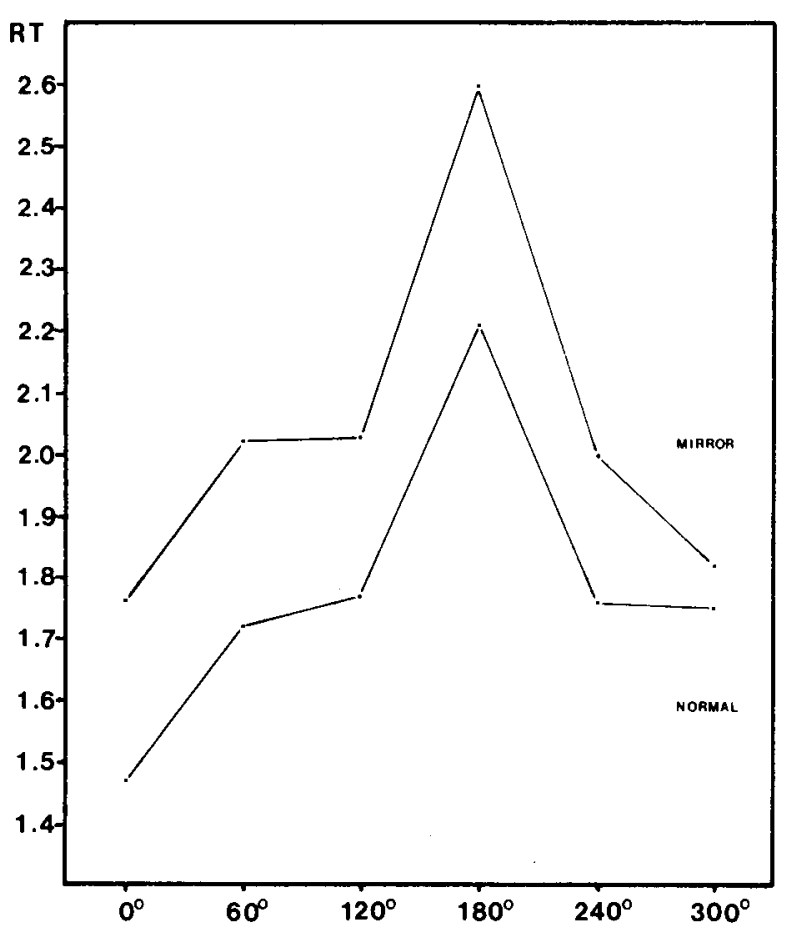

Figure 3. Mean verification response times (in seconds) pooled over Blocks 2-5 for each orientation angle.

\section{CONCLUSIONS}

Corballis et al. (1978) found that with small numbers of Roman capitals and Arabic digits, the identification of a presented symbol was, first, independent of the orientation of that symbol and, second, likely the result of the extraction of critical features encoded in a fashion invariant to orientation. Symbol identification, in contrast to symbol verification (e.g., Cooper \& Shepard, 1973), was not found to involve mental rotation processes.

In the present research, it was argued that the use of either a feature extraction or a mental rotation strategy of symbol identification could conceivably be a function of both a subject's familiarity with the symbol and the range of possibilities of which the symbol was a member. The more novel a symbol was and the larger was the set of symbols from which a presented symbol was sampled, the greater should be the processing demands attendant on encoding, maintaining, and retrieving representations of critical features that were orientation invariant. It seemed feasible that at some level of novelty, and with some size of symbol set, these processing demands might prompt a switch to a mental rotation strategy. In that the Corballis et al. (1978) experiments had employed small numbers of highly overlearned alphanumeric symbols, it was argued that such a strategy. switching possibility had not been explicitly tested. The present research sought to make such a test.

In Experiment 1, symbol familiarity was manipu- lated by varying the criterion to which subjects were trained in learning to label novel letter-like symbols. Set size was manipulated by varying the number of these symbols used in later identification trials. It was found that response times to identify rotated symbols were constant across nonstandard orientations, regardless of both familiarity and set size. There was no suggestion of any increases in response times with increased deviation in symbol orientation away from the standard upright. These findings were thus interpreted in terms of subjects' having employed feature extraction processes in identifying the symbols. It would seem that the Corballis et al. (1978) findings are generalizable to symbols other than highly overlearned alphanumeric symbols and to sample sets of larger numbers. This interpretation was further supported by the Experiment 2 findings, which replicated Cooper and Shepard's (1973) mental rotation findings with the verification of rotated symbols, but with the novel symbols used in Experiment 1.

In conclusion, the present research suggests a very interesting question for future study. Was the lack of a mental rotation strategy of identification evidenced here simply the result of insufficient variations being made in familiarity and set size? Or rather, is the reason more fundamental? Is it possible that symbol identification is basically a function of feature extraction processes, with variations of things like symbol orientation, symbol familiarity, and symbol set size having effects on the relative efficiency of processing but not on the fundamental nature of that processing? The present research would at least suggest that such a possibility is worthy of future investigation.

\section{RATLIJNCE NOTE}

1. Cooper, L. A. Spatial information processing: Strategies for research. Paper presented at the ONR/NPRDC conference on "Aptitude, Learning, and Instruction: Cognitive Process Analysis," San Diego, Calif., March 6-9, 1978.

\section{REFERINCES}

ArCher, E. J. A re-evaluation of the meaningfulness of all possible CVC trigrams. Psychological Monographs, 1960, 74(10, Whole No. 497).

COOPER, L. A. Individual differences in visual comparison processes. Perception \& Psychophysics, 1976, 19, 433-44.

Cooper, L. A., \& Podoonny, P. Mental transformations and visual comparison processes: Effects of complexity and similarity. Journal of Experimental Psychology: Human Perception and Performance, 1976, 2, 503-514.

Cooper, L. A., \& Shepard, R. N. Chronometric studies of the rotation of mental images. In W. G. Chase (Ed.), Visual information processing. New York: Academic Press, 1973.

Conballis, M. C., Zbrodort, N. J., Shetren, L. I., \&utuen, P. B. Decisions about identity and orientation of rotated letters and digits. Memory Cognition, 1978, 6, , 98-107.

EteY, M. G. Distinguishing imagery from propositional recoding processes in sentence-picture verification tasks. Canadian Journal of Psychology, 1981, 35, 254-269.

Gibson, E. J., Gibson, J. J., Pick, A. D., \& Osgen, H. A. A 
developmental study of the discrimination of letter-like forms. Journal of Comparative and Physiological Psychology, 1962, 55, 897-906.

Glushro, R. J., \& Cooper, L. A. Spatial comprehension and comparison processes in verification tasks. Cognitive Psychology, 1978, 10, 391-421.

Kosslyn, S. M., Murphy, G. L., Bemesderfer, M. E., \& Feingtein, K. J. Category and continuum in mental comparisons. Journal of Experimental Psychology: General, 1977, 106, 341-375.

Petrusic, W. M., Varro, L., \& Jamieson, D. G. Mental rota- tion validation of two spatial ability tests. Psychological Research, 1978, 40, 139-148.

PYlYshyn, Z. W. The rate of "mental rotation" of images: A test of a holistic analogue hypothesis. Memory \& Cognition, 1979, 7, 19-28.

TAPLey, S. M., \& Bryden, M. P. An investigation of sex differences in spatial ability: Mental rotation of three-dimensional objects. Canadian Journal of Psychology, 1977, 31, 122-130.

(Received for publication February 13, 1981; revision accepted May 28, 1981.) 